\title{
Inhibition of LSD1 phosphorylation alleviates colitis symptoms induced by dextran sulfate sodium
}

\author{
Chaeyoon $\mathrm{Oh}^{1}$, Jiyeong Jeong ${ }^{2,3}$, Se Kyu $\mathrm{Oh}^{4}$, Sung Hee Baek ${ }^{4}$ \& Keun Il Kim ${ }^{1,2, *}$ \\ ${ }^{1}$ Department of Biological Sciences, Cellular Heterogeneity Research Center, Sookmyung Women's University, Seoul 04310, ${ }^{2}$ Research \\ Institute of Women's Health, Sookmyung Women's University, Seoul 04310, ${ }^{3}$ Division of Pulmonary and Critical Care Medicine, \\ Department of Internal Medicine, Seoul National University Hospital, Seoul 03080, ${ }^{4}$ Department of Biological Sciences, Creative Research \\ Initiative Center for Chromatin Dynamics, Seoul National University, Seoul 08826, Korea
}

\begin{abstract}
Inflammatory Bowel Disease is caused by an acute or chronic dysfunction of the mucosal inflammatory system in the intestinal tract. In line with the results of our previous study, wherein we found that the PKC $\alpha$-LSD1-NF-KB signaling plays a critical role in the prolonged activation of the inflammatory response, we aimed to investigate the effect of signaling on colitis in the present study. Lsd1 S112A knock-in ( $L s d 1^{S A / S A}$ ) mice, harboring a deficiency in phosphorylation by $\mathrm{PKC} \alpha$, exhibited less severe colitis symptoms and a relatively intact colonic epithelial lining in dextran sulfate sodium (DSS)induced colitis models. Additionally, a reduction in pro-inflammatory gene expression and immune cell recruitment into damaged colon tissues in $L S d 1^{S A / S A}$ mice was observed upon DSS administration. Furthermore, LSD1 inhibition alleviated colitis symptoms and reduced colonic inflammatory responses. Both LSD1 phosphorylation and its activity jointly play a role in the progression of DSS-induced colitis. Therefore, the inhibition of LSD1 activity could potentially protect against the colonic inflammatory response. [BMB Reports 2020; 53(7): 385-390]
\end{abstract}

\section{INTRODUCTION}

Inflammatory Bowel Disease (IBD) is an idiopathic and relapsing inflammatory disorder occurring in the gastrointestinal (Gl) tract, and has two major subtypes, namely Crohn's disease and ulcerative colitis $(1,2)$. Despite symptom similarity, Crohn's disease and ulcerative colitis show distinctive characteristics in damaged regions, pro-inflammatory cytokine profiles and his-

*Corresponding author. Tel: +82-2-710-9768; Fax: +82-2-20777322; E-mail: kikim@sookmyung.ac.kr

https://doi.org/10.5483/BMBRep.2020.53.7.298

Received 29 November 2019, Revised 23 December 2019, Accepted 16 January 2020

Keywords: Colitis, Dextran sulfate sodium (DSS), Inflammatory bowel disease (IBD), Inflammation, LSD1 tological phenotypes $(1,2)$. Crohn's disease shows a non-continuous damaged region in the Gl tract (3-5). Conversely, ulcerative colitis involves an extensive area, from the proximal to distal colon, in a continuous fashion (3-5). Various animal models have become useful tools to investigate the mediator of the mucosal immune response and immunological processes underlying acute or chronic intestinal inflammation, improving the understanding of $\operatorname{IBD}(6,7)$. The dextran sulfate sodium (DSS)-induced colitis model is the most widely used in IBD research, even though most IBD experimental models are not appropriate due to disease complexity $(8,9)$.

DSS is a negatively charged sulfated polysaccharide that can be easily and rapidly administered as a water-soluble agent $(10,11)$. Although the exact mechanism underlying colitis induction by DSS is unclear, the administration of DSS solution damages the epithelial monolayer cells and mucosal layer in the large intestine. Consequently, gut microbiota enter the colonic lumen and induce a radical immunity imbalance (9). Massive pro-inflammatory cytokines, such as IL-6, IL-1 $\beta$, and TNF- $\alpha$, are also released in mouse colon tissues with DSSinduced acute or chronic colitis $(6,12)$. Therefore, pro-inflammatory cytokines have become a useful maker for colitis in animal models.

Nuclear factor- $\kappa \mathrm{B}(\mathrm{NF}-\kappa \mathrm{B})$ plays a key role in the regulation of intestinal inflammation and colitis-associated cancer (13-15). The aberrant regulations of canonical and non-canonical NF- $\mathrm{KB}$ signaling have been well studied in IBD patients (13-15). This condition induces the release of vast amounts of pro-inflammatory cytokines, such as TNF- $\alpha$, IL-1 $\beta$, and IL- 6 , which induces tissue damage and immune cell infiltration $(16,17)$. Posttranslational modification of NF- $\mathrm{KB}$ by LSD1, NSD1/FBXL11, and PRMT5 is associated with its diverse functions (18-20).

Lysine-specific demethylase 1 (LSD1, also called KDM1A) catalyzes the demethylation of histone $\mathrm{H} 3$ (21). H3K4me2 is generally associated with gene activation, while $\mathrm{H} 3 \mathrm{~K} 9 \mathrm{me} 2$ is associated with gene suppression. Thus, LSD1 acts as a transcriptional activator or inhibitor, depending on the residues of the target histone $\mathrm{H} 3$ (22-24). In addition to its well-known $\mathrm{H} 3$ demethylase function, LSD1 also demethylates non-histone proteins, including p53, HIF-1 $\alpha$, and the $\mathrm{p} 65$ subunit of NF- $\mathrm{KB}$

ISSN: 1976-670X (electronic edition)

Copyright (c) 2020 by the The Korean Society for Biochemistry and Molecular Biology

(c) This is an open-access article distributed under the terms of the Creative Commons Attribution Non-Commercial License (http://creativecommons.org/licenses/by-nc/4.0) which permits unrestricted non-commercial use, distribution, and reproduction in any medium, provided the original work is properly cited. 
(25-27). LSD1 cellular function is controlled by phosphorylation, mediated by several kinases including PLK1, CK2/WIP1, and PKC $\alpha(18,28,29)$. Particularly, LSD1 phosphorylation on the $112^{\text {th }}$ serine residue in mice (analogous to $111^{\text {th }}$ serine in human LSD1) by PKC $\alpha$, was identified as a critical regulatory point for LSD1 functioning in circadian rhythm and inflammatory signaling $(18,30)$. In inflammatory signaling, lipopolysaccharide (LPS) stimulation of immune cells like macrophages activates $\mathrm{PKC} \alpha$, which then phosphorylates LSD1 on serine$112(18,28,29)$. Although LSD1 phosphorylation does not alter demethylase activity, it modulates interaction of LSD1 with substrate (18). Phosphorylated LSD1 interacts with monomethylated p65 and removes the methyl group, leading to the

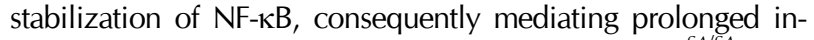
flammatory gene expression (18). Subsequently, $L s d 1^{S A / S A}$ mice harboring substitution of $112^{\text {th }}$ serine to alanine, exhibited alleviated symptoms than wild-type mice in the sepsis model (18).

We investigate whether the suppression of the LSD1-NF-kB pathway could reduce colitis and whether an LSD1 inhibitor could alleviate ulcerative colitis symptoms in mice. Consequently, we found that both lack of LSD1 phosphorylation and inhibition of LSD1 activity reduced colitis symptoms, infiltration of immune cells, and pro-inflammatory gene expression in mice.

\section{RESULTS}

\section{Lack of LSD1 phosphorylation alleviates the symptoms of DSS-induced acute colitis}

Our previous study revealed the involvement of LSD1 phosphorylation on $112^{\text {th }}$ serine residue in the prolonged activation of the NF-kB (18). Here, we investigated whether the phosphorylation plays a role in colitis (where uncontrolled inflammation is the main cause of the disease) using a DSSinduced colitis mouse model. $L s d 1^{+/+}$and $L s d 1^{S A / S A}$ mice were administered a $2 \%$ DSS solution for 5 days (injury phase), followed by normal drinking water for 5 days (recovery phase) (Fig. 1A), which is a modified protocol from Wirtz et al. (31). $L s d 1^{+/+}$mice showed progressive weight loss up to 10 days after DSS administration, reaching about $80 \%$ of their original body weight. Whereas $L s d 1^{S A / S A}$ mice recovered earlier from body weight loss, while exhibiting much milder body weight loss than $L s d 1^{+/+}$mice (Fig. 1B). Disease activity index (DAI) scores increased during DSS treatment in both genotypes, but started to decrease earlier in $L s d 1^{S A / S A}$ than in $L s d 1^{+/+}$mice (Fig. 1C), indicating that $L s d 1^{S A / S A}$ mice are less susceptible to DSS-induced colitis compared to their wild-type control.

We then obtained the colon tissues from four groups of mice $\left(L s d 1^{+/+}\right.$versus $L s d 1^{S A / S A}$ mice, with and without DSS administration) at 10 days from the start of DSS administration and examined the tissue appearance. Colon length was similar in the absence of DSS administration, in both $L s d 1^{+/+}$and $L s d 1^{\text {SAISA }}$ mice. DSS administration induced colon length shortening in both genotypes, however, the phenotype was less prominent in $L s d 1^{S A / S A}$ mice compared to $L s d 1^{+++}$mice (Fig.
1D and 1E). The expression of $\mathrm{Ki}-67$ and proliferating cell nuclear antigen (PCNA), marker proteins of cell proliferation, were increased in $\mathrm{Lsd1}^{+/+}$colon tissues during DSS administration, whereas the increase was not significant in $L s d 1^{S A / S A}$ colon tissues (Fig. 1F and 1G). These results indicate that the increased cell proliferation in $\mathrm{Lsd1}^{+/+}$colonic epithelium restores epithelium from DSS-induced damage, and that the reduced increase in $\mathrm{Ki}-67$ and PCNA expression represents less damage in $L s d 1^{S A / S A}$ mice. Considered collectively, these data suggest that a lack of LSD1 phosphorylation on $112^{\text {th }}$ serine residue alleviates the symptoms of DSS-induced colitis in mice.

\section{$L s d 1^{S A / S A}$ mice exhibit a reduced colonic inflammatory response upon DSS administration}

We then subjected the colon tissues, which had undergone the injury and recovery phases, to hematoxylin \& eosin (H\&E) staining and examined the detailed histological features. Most
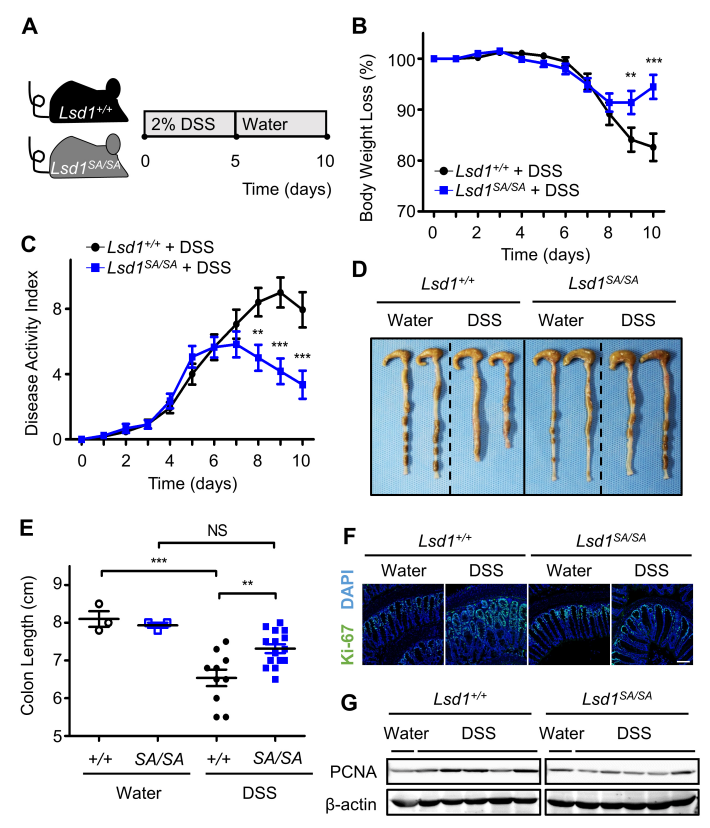

Fig. 1. Susceptibility analyses for DSS-induced colitis by comparing $L s d 1^{+/+}$and $L s d 1^{S A S A}$ mice. (A) Schematic diagram of the DSSinduced colitis model. Male mice received $2 \%$ DSS in drinking water for 5 days, followed by normal drinking water for 5 days. (B) Body weight loss was measured relative to the initial weight ( $\mathrm{n}=17$ per group). (C) The DAl of colitis was monitored daily ( $\mathrm{n}=$ 17 per group). (D) Representative pictures of the colon. Colon tissue from each group was obtained 10 days after the first DSS treatment. (E) Graph of colon length. Each symbol represents an individual mouse from each group. (F) Immunofluorescence staining of Ki-67 in colon tissues. Nuclei were stained with DAPI. Scale bar $=100 \mu \mathrm{m}$. (G) Immunoblot analysis of PCNA in colons from $L s d 1^{+/+}$and $L s d 1^{\mathrm{SASA}}$ mice. $\beta$-actin is an equal loading control. Each lane represents a mouse. $* * P<0.01, * * * P<0.001$ for whole figures. 
of the colonic epithelium of $L s d 1^{+/+}$mice were severely damaged and disrupted from DSS administration. Whereas the colon of $L s d 1^{S A S A}$ mice showed less damaged and relatively intact epithelial structure compared to that of $\mathrm{Lsd1}^{+/+}$mice (Fig. 2A). Furthermore, histopathological score, measured by macroscopic images of H\&E stained whole colonic sections, was also lower in $L s d 1^{S A / S A}$ mice (Fig. 2B). Therefore, these data imply that $L s d 1^{S A / S A}$ mice exert a protective effect against the intestinal epithelial damage induced by DSS administration in colon tissue. To evaluate the extent of the inflammatory response in the colon, we measured the mRNA levels of proinflammatory cytokines. The mRNA levels of the $I I-1 \beta, \| l-6$, and II-8 genes in colon tissues were similar between $\mathrm{Lsd} 1^{+/+}$ and $L s d 1^{S A / S A}$ mice from the control group (without DSS administration) and increased two- to five-fold in $\mathrm{Lsd}^{+/+}$mice with DSS administration (Fig. 2C). However, the increase in mRNA levels of all three genes, during DSS administration in $L s d 1^{\text {SAISA }}$ mice, was relatively low compared to the control

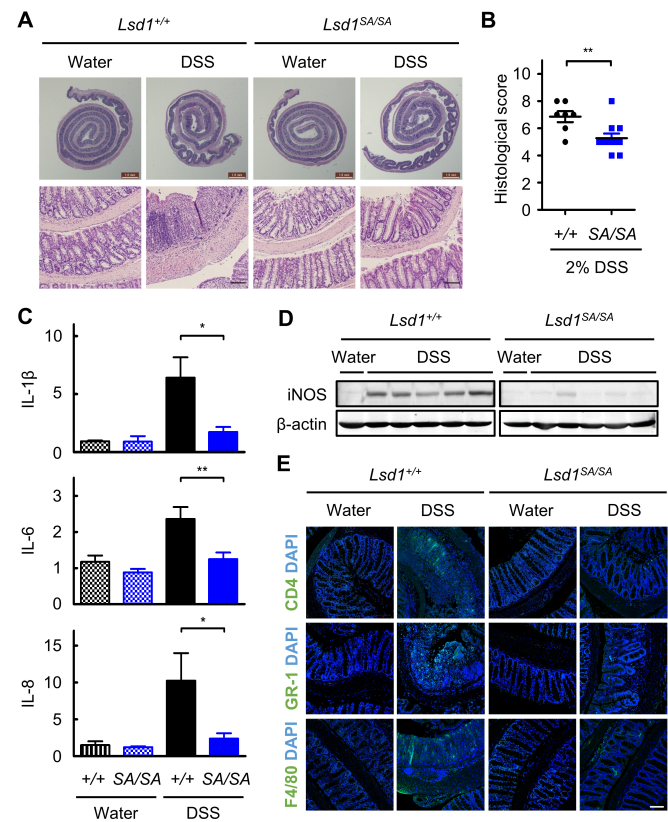

Fig. 2. Analysis of histology and inflammatory response of $L s d 1^{+/+}$ and $L s d 1^{S A / S A}$ mice in DSS-induced colitis. (A) Microscopic images of $\mathrm{H} \& \mathrm{E}$ stained colonic sections. Scale bars, $1 \mathrm{~mm}$ (upper panel) and $200 \mu \mathrm{m}$ (lower panel). (B) Histological scores were determined based on the mucosal hyperemia, surface epithelium loss, and crypt damage. $* * P<0.01$. Each symbol represents an individual mouse from each group. (C) RT-qPCR data for the expression of proinflammatory $I I-1 \beta, \quad I I-6$, and $I I-8$ genes. ( $L s d 1^{++}$and $L s d 1^{\text {SAISA }}$ with water, $\mathrm{n}=3 ; \mathrm{Lsd}^{+/+}$with DSS, $\mathrm{n}=5$; and $L s d 1^{\text {SASSA }}$ with DSS, $\mathrm{n}=7$ ), $* \mathrm{P}<0.05, * * \mathrm{P}<0.01$. (D) Immunoblot analysis of iNOS in colons from $L s d 1^{+/+}$and $L s d 1^{S A / S A}$ mice. $\beta$-actin is an equal loading control. Each lane represents a mouse. (E) Immunofluorescence staining for CD4, GR-1, and F4/80 in colon tissues. Nuclei were stained with DAPI. Scale bar $=100 \mu \mathrm{m}$. group (Fig. 2C). Additionally, the level of iNOS proteins induced by DSS administration was significantly reduced in $L s d 1^{S A / S A}$ mice, compared to that of $L s d 1^{+/+}$mice (Fig. 2D). As additional criteria of the inflammatory response, we examined the infiltration of immune cells in the colon tissues by staining the sections with antibodies against CD4 (T cell), Gr-1 (neutrophil), and F4/80 (macrophage). Fluorescence signals from all three antibodies were clearly detected in the immunofluorescence data of $\mathrm{Lsd}^{+/+}$colon tissues, indicating infiltration of these immune cells to the colon tissue (Fig. 2E). Colon tissues of $L s d 1^{S A / S A}$ mice showed much less fluorescence signals from all three antibodies. Collectively, these data suggest that $L s d 1^{S A / S A}$ mice are less susceptible to epithelial damage of the colon tissues, induced by DSS administration, with a reduced inflammatory response.

\section{Inhibition of LSD1 activity alleviates colitis symptom by reducing inflammatory responses in mouse colon tissue} We then used GSK-LSD1, a demethylase inhibitor of LSD1, to investigate whether the inhibition of LSD1 activity could alleviate colitis symptoms. GSK-LSD1 ( $5 \mathrm{mg} / \mathrm{kg}$ body weight) was injected intraperitoneally on days 0,1 , and 3 of DSS administration (Fig. 3A). The control group was injected with the same volume of PBS instead of GSK-LSD1. The GSK-LSD1 injection group showed milder symptoms than the PBS group 8 days after acute colitis induction, based on body weight recovery and DAl scores (Fig. 3B and 3C). Colon length shortening was also less severe in the GSK-LSD1 injection group compared to the PBS control (Fig. 3D and 3E). Consistent with the less severe colitis symptoms, Ki-67 expression (representing cell proliferation in colonic epithelium) decreased in the GSK-LSD1 injection group (Fig. 3F). Thus, these results demonstrate the protective effect of LSD1 inhibition in mice, against DSS-induced acute colitis.

Considering that GSK-LSD1 injections produced similar results to $L s d 1^{S A / S A}$ mice, colon tissues were subjected to a histological analysis. DSS-administration almost completely destroyed the crypt structure of the colonic epithelium, whereas GSK-LSD1 injection protected colon tissues from damage, resulting in significantly intact crypt structures (Fig. 4A). Histological score also revealed less severe mucosal hyperemia and tissue damage in the GSK-LSD1 injection group, compared to the control group (Fig. 4B). Additionally, we identified a decrease in mRNA levels of proinflammatory cytokines and in immune cell infiltration levels in the GSKLSD1 injection group, as a measure of the degree of inflammatory response (Fig. 4C and 4D). In summary, DSS destroys the protective barrier of the colonic epithelium, causing an inflammatory response that induces colitis. The absence of LSD1 phosphorylation in $L s d 1^{S A / S A}$ mice, or the injection of an LSD1 inhibitor in wild-type mice, reduced the degree of inflammatory responses, which attenuates the progression of colitis due to DSS (Fig. 4E). 

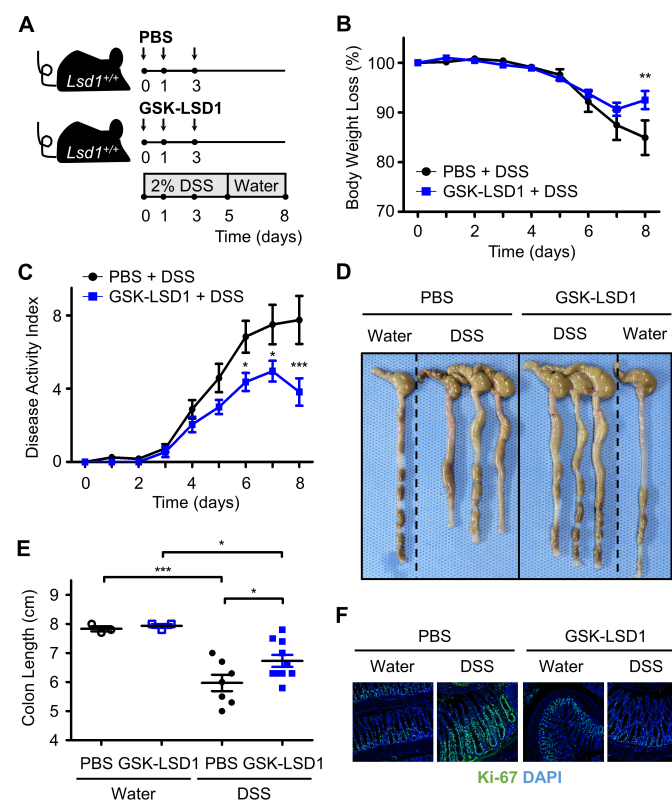

D

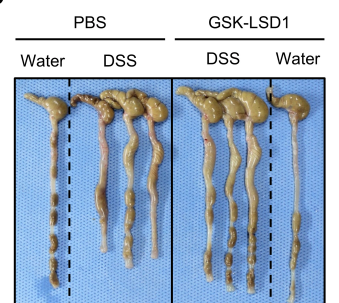

$\mathbf{F}$

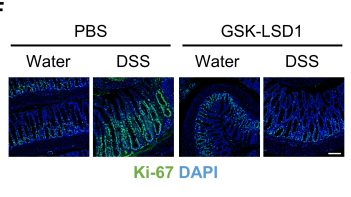

Fig. 3. Effect of GSK-LSD1 on alleviating DSS-induced colitis symptoms. (A) Schematic diagram of a DSS-induced colitis model. All groups were intraperitoneally injected with $5 \mathrm{mg} / \mathrm{kg}$ GSK-LSD1 or $\mathrm{PBS}$, in equal volumes, on days 0,1 , and 3. (B) Body weight loss of mice was measured relative to initial weight. (C) The DAI of colitis was monitored daily. Statistical analysis used two-way ANOVA (PBS + DSS, $\mathrm{n}=12$; GSK-LSD1 + DSS, $\mathrm{n}=11$ ), ${ }^{*} \mathrm{P}<$ $0.05, * * * P<0.001$. (D) Colon tissues of each group were obtained 8 days after the first DSS treatment. (E) Graph of colon length. ${ }^{*} P<0.05,{ }^{*} * \mathrm{P}<0.001$. Each symbol represents an individual mouse from each group. (F) Immunofluorescence staining of $\mathrm{Ki}-67$ in colon tissues. Nuclei were stained with DAPI. Scale bar $=100 \mu \mathrm{m}$.

\section{DISCUSSION}

Uncontrolled inflammation is a well-known trigger of human $\operatorname{IBD}(7,8)$. In this study, we observed the relief of colitis symptoms along with the downregulation of the inflammatory response in $L s d 1^{S A / S A}$ mice and $L s d 1^{++}$mice injected with GSK-LSD1. These results indicate that LSD1 phosphorylation and the demethylase activity of LSD1 participate in the progression of inflammatory responses in the experimental colitis model. Thus, the inhibition of LSD1 phosphorylation or LSD1 activity may be utilized to control human IBD.

Recent studies indicated that LSD1 phosphorylation on $112^{\text {th }}$ serine $\left(111^{\text {th }}\right.$ in human) is crucial for a variety of functions, including epithelial-mesenchymal transition (EMT), cancer metastasis, circadian rhythmicity and inflammation (18, 30, 32, 33). Ectopic expression of the LSD1 S111D mutant, mimicking the phosphorylated form of LSD1, promoted the metastatic capacity of MDA-MB-231 breast cancer cells in nude mice (32). Additionally, PKCO-mediated phosphorylation of LSD1

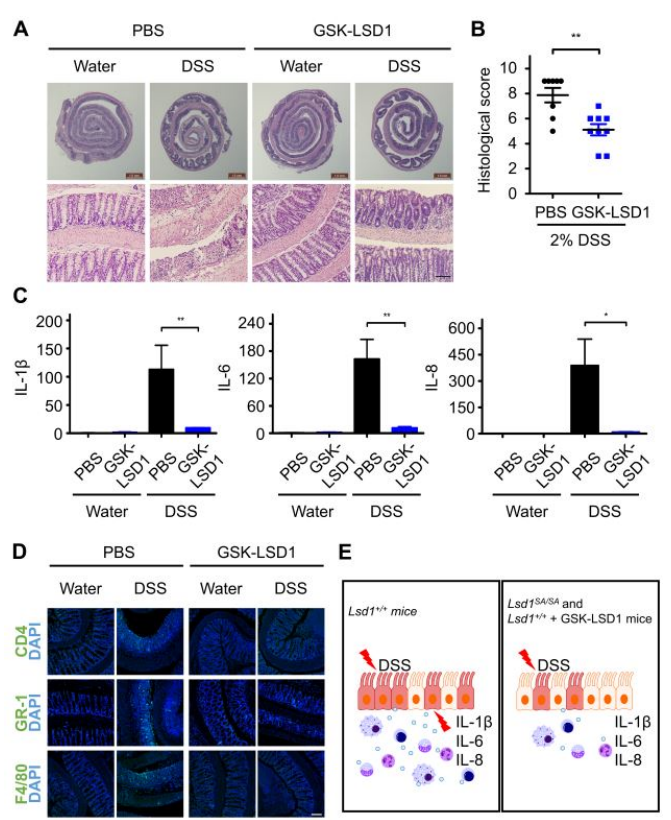

Fig. 4. Effect of GSK-LSD1 on the inflammatory response in DSSinduced colitis. (A) Microscopic images of $\mathrm{H} \& \mathrm{E}$ stained colonic sections. Scale bars, $1 \mathrm{~mm}$ (upper panel) and $200 \mu \mathrm{m}$ (lower panel). (B) Histological scores were measured based on the mucosal hyperemia, surface epithelium loss, and crypt damage. $* * P<$ 0.01 . Each symbol represents an individual mouse from each group. (C) The amount of pro-inflammatory cytokines $I /-1 \beta$, II-6, and II-8 was analyzed with RT-qPCR, 8 days after the start of the experiment. ${ }^{*} \mathrm{P}<0.05$ and $* * \mathrm{P}<0.01$, ( $\mathrm{n}=3$ per group). (D) Immunofluorescence staining of CD4, GR-1, and F4/80 in colon tissues. Nuclei were stained with DAPI. Scale bar $=100 \mu \mathrm{m}$. (E) Graphical overview of this study.

on the residue was critical for its demethylase and EMT promoting activity in breast cancer (33). PKC $\alpha$ is the first enzyme identified as a kinase, responsible for LSD1 phosphorylation on the residue, in the regulation of the circadian cycle (30). LSD1 phosphorylation by PKC $\alpha$ is also critical for the prolonged activation of NF- $\mathrm{KB}$ signaling (18). Intestinal epithelial cells express at least 10 PKC isoforms; not only "classic" PKC isoforms like PKC $\alpha$, but also "novel" PKC isoenzymes like PKC $\theta$ (34). Although PKC $\alpha$ phosphorylated LSD1 upon LPS stimulation (18), DSS-induced colitis is a complex process involving a number of proinflammatory agents. We therefore cannot rule out the possibility that LSD1 is phosphorylated by PKC $\alpha$ as well as other kinases, including PKC $\theta$, in colitis models.

In the previous study we successfully used GSK-LSD1, an inhibitor of LSD1 demethylase activity, to reduce systemic inflammation induced by LPS or CLP (cecal ligation and puncture) (18). The demethylase activity of LSD1 is required for demethylation of methyl-p65, a subunit of the NF- $\mathrm{BB}$ transcription factor, resulting in the stabilization of p65 and 
prolonged activation of NF-кB. Inhibition of LSD1 by GSKLSD1 resulted in the accumulation of mono-methylated p65, which was eventually degraded by the ubiquitin-proteasome system resulting in premature termination of the NF-кBmediated inflammatory responses. In this study, we identified the beneficial effect of GSK-LSD1 on the DSS-induced colitis mouse model. Relief of colitis symptoms was similar to what we saw in $L s d 1^{S A / S A}$ mice. Since $L s d 1^{S A / S A}$ is a whole-body knock-in mouse, it is not possible to pinpoint which cell type (i.e., colonic epithelial cells or immune cells) is involved in alleviating colitis symptoms through the lack of LSD1 phosphorylation or LSD1 inhibition. Since cytokine crosstalk between immune and epithelial cells affects colitis, the suppression of inflammatory responses in both cell types, through the inhibition of LSD1 phosphorylation or its activity, most likely contributes to the relief of colitis symptoms.

Currently, LSD1 is considered a validated epigenetic target for anticancer drug development, with many LSD1 inhibitors in clinical trials as anticancer agents for AML (acute myeloid leukemia), SCLC (small-cell-lung cancer) and other solid tumors $(35,36)$. In this study, we showed the potential of LSD1 inhibitors as anti-inflammatory agents that can alleviate colitis symptoms. Since high levels of LSD1 expression have been identified in inflammation-associated cancers, including colorectal cancer (36), the LSD1 inhibitor, GSK-LSD1, has therapeutic potential in colitis and may be good candidates for limiting colitis-associated cancer (CAC) progression, by targeting the microenvironment as well as the cancer itself.

\section{MATERIALS AND METHODS}

\section{Mice}

Generation of $L s d 1^{S A / S A}$ mice has been described previously (30). Mice were handled in accordance with the animal handling guidelines of Sookmyung Women's University. Procedures were approved by the Institutional Animal Care and Use Committee of the University (SMWU-IACUC-1711-029).

\section{DSS-induced colitis}

Male mice with corresponding age (7-9 weeks) and body weight (20-25 g) received 2\% DSS (MP Biomedicals, M.W. 36-50 kDa) in drinking water for 5 days, followed by normal drinking water for 5 days. In the LSD1 inhibition groups, wild-type mice were intraperitoneally injected with the LSD1 inhibitors, GSK-LSD1 (5 mg/kg body weight, Sigma) or PBS, on days 0,1 , and 3 of $2 \%$ DSS administration. Control mice received DSS-free drinking water. The DAI of colitis was monitored daily, based on Supplementary Table 1.

\section{Hematoxylin \& Eosin (H\&E) and immunofluorescence staining}

Mouse colon tissues were fixed in a $4 \%$ paraformaldehyde solution at $4^{\circ} \mathrm{C}$ for 2 days and embedded in paraffin. All samples were cut into $4 \mu \mathrm{m}$ sections for H\&E staining. Micro- scopic images were obtained through light microscopy (Olympus IX71 and Leica M80). Histological scoring was based on three categories, namely, mucosal hyperemia, surface epithelium loss, and crypt damage (Supplementary Table 2). Immune cell infiltration into colon epithelium was analyzed with immunofluorescence staining. The primary antibodies used were anti-Ki67 (Abcam, ab15580), anti-CD4 (BD Biosciences, \#553047), anti-Gr-1 (Serotech, \#MCA2387GA), and anti-F4/80 (Serotech, \#MCA497GA).

\section{Quantitative RT-PCR of mRNAs from colon tissues}

Mouse colon tissues were homogenized in a Trizol reagent (Life Technologies) and total RNA was isolated using the GeneJET RNA Purification Kit (Thermo Fisher Scientific). Complementary DNA (cDNA) was synthesized from $1 \mu \mathrm{g}$ of total RNA using the cDNA synthesis kit (Thermo Fisher Scientific). Primers used for mouse genes are in Supplementary Table 3.

\section{Westem blotting}

Colon tissues were washed with ice-cold PBS and solution $\mathrm{B}$ (2.7 $\mathrm{mM} \mathrm{KCl}, 150 \mathrm{mM} \mathrm{NaCl}, 1.2 \mathrm{mM} \mathrm{KH} \mathrm{PO}_{4}, 680 \mathrm{mM}$ $\mathrm{Na}_{2} \mathrm{HPO}_{4}, 1.5 \mathrm{mM}$ EDTA, and $1 \mathrm{mM}$ DTT). Fragments of the distal portion were then homogenized in RIPA buffer $(150 \mathrm{mM}$ $\mathrm{NaCl}, 20 \mathrm{mM}$ Tris $\mathrm{pH}$ 7.5, 0.1\% SDS, 1\% NP-40, and $2 \mathrm{mM}$ EDTA). Proteins were resolved on $8-15 \%$ gradient gels and transferred onto a nitrocellulose membrane (GE Healthcare). The primary antibodies used were anti- $\beta$-actin (AB Frontier), anti-iNOS (Merck Millipore), and anti-PCNA (Abcam).

\section{Statistical analysis}

Statistical analyses were performed with GraphPad Prism v5.01 software. The statistical significance of the two groups was analyzed by two-way ANOVA (Body weight and DAI) or Mann-Whitney test (Histological score). One-way ANOVA (Newman keule's test) was used for the statistical analysis among four groups. Data were expressed as the mean \pm SEM.

\section{ACKNOWLEDGEMENTS}

This work was supported by the Science Research Center Program (Cellular Heterogeneity Research Center, NRF-2016 R1A5A1011974) and by Basic Science Research Program (NRF-2018R1A2B6004112) to K.I.K. through the National Research Foundation of Korea (NRF) funded by the Ministry of Science and ICT. We would like to thank Editage (www. editage.co.kr) for English language editing.

\section{CONFLICTS OF INTEREST}

The authors have no conflicting interests.

\section{REFERENCES}

1. Podolsky DK (2002) Inflammatory bowel disease. N Engl J 
Med 347, 417-429

2. Kostić M, Djakovic L, Šujić R, Godman B and Janković SM (2016) Inflammatory bowel diseases (crohn's disease and ulcerative colitis): cost of treatment in serbia and the implications. Appl Health Econ Health Policy 15, 85-93

3. Kang I, Lee BC, Lee JY et al (2019) Interferon- $\gamma$-mediated secretion of tryptophanyl-tRNA synthetases has a role in protection of human umbilical cord blood-derived mesenchymal stem cells against experimental colitis. BMB Rep 52, 318-323

4. Yu YR and Rodriguez JR (2017) Clinical presentation of crohn's, ulcerative colitis, and indeterminate colitis: Symptoms, extraintestinal manifestations, and disease phenotypes. Semin Pediatr Surg 26, 349-355

5. Oliva-Hemker M, Carvalho RS, Cuffari C, Abadom V, Dilworth HP and Thompson R (2006) Indeterminate colitis: A significant subgroup of pediatric IBD. Inflamm Bowel Dis 12, 258-262

6. Alex P, Zachos NC, Nguyen T et al (2009) Distinct cytokine patterns identified from multiplex profiles of murine DSS and TNBS-Induced colitis. Inflamm Bowel Dis $15,341-352$

7. Strober W, Fuss IJ and Blumberg RS (2002) The immunology of mucosal models of inflammation. Annu Rev Immunol 20, 495-549

8. Khor B, Gardet A and Xavier RJ (2011) Genetics and pathogenesis of inflammatory bowel disease. Nature 474, 307-317

9. Chassaing B, Aitken JD, Malleshappa M and Vijay-Kumar M (2014) Dextran sulfate sodium (DSS)-induced colitis in mice. Curr Protoc Immunol 104, 15.25.11-15.25.14

10. Okayasu I, Hatakeyama S, Yamada M, Ohkusa T, Inagaki $\mathrm{Y}$ and Nakaya R (1990) A novel method in the induction of reliable experimental acute and chronic ulcerative colitis in mice. Gastroenterology 98, 694-702

11. Wirtz S, Neufert C, Weigmann B and Neurath MF (2007) Chemically induced mouse models of intestinal inflammation. Nat Protoc 2, 541-546

12. Nakamura $M$, Saito $H$, Kasanuki J, Tamura $Y$ and Yoshida $S$ (1992) Cytokine production in patients with inflammatory bowel disease. Gut 33, 933-937

13. Atreya I, Atreya R and Neurath MF (2008) NF- $\kappa B$ in inflammatory bowel disease. J Intern Med 263, 591-596

14. Schottelius AJ and Dinter H (2006) Cytokines, NF-кB, microenvironment, intestinal inflammation and cancer. Cancer Treat Res 130, 67-87

15. Viennois E, Chen F and Merlin D (2013) NF-kB pathway in colitis-associated cancers. Transl Gastrointest Cancer 2, 21-29

16. McDaniel DK, Eden K, Ringel VM and Allen IC (2016) Emerging roles for noncanonical NF- $\kappa B$ signaling in the modulation of inflammatory bowel disease pathobiology. Inflamm Bowel Dis 22, 2265-2279

17. Neurath MF, Fuss I, Schurmann G et al (1998) Cytokine gene transcription by NF- $\mathrm{KB}$ family members in patients with inflammatory bowel disease. Ann N Y Acad Sci 859, 149-159

18. Kim D, Nam HJ, Lee W et al (2018) PKC $\alpha$-LSD1-NF-кBsignaling cascade is crucial for epigenetic control of the inflammatory response. Mol Cell 69, 398-411.e396

19. Lu T, Jackson MW, Wang B et al (2009) Regulation of NF- $\kappa B$ by NSD1/FBXL11-dependent reversible lysine methylation of p65. Proc Natl Acad Sci U S A 107, 46-51

20. Wei H, Wang B, Miyagi M et al (2013) PRMT5 dimethylates R30 of the p65 subunit to activate NF-kB. Proc Natl Acad Sci U S A 110, 13516-13521

21. Culhane JC and Cole PA (2007) LSD1 and the chemistry of histone demethylation. Curr Opin Chem Biol 11, 561-568

22. Shi Y, Lan F, Matson C et al (2004) Histone demethylation mediated by the nuclear amine oxidase homolog LSD1. Cell 119, 941-953

23. Forneris F, Binda C, Dall'Aglio A, Fraaije MW, Battaglioli E and Mattevi A (2006) A highly specific mechanism of histone $\mathrm{H} 3-\mathrm{K} 4$ recognition by histone demethylase LSD1. J Biol Chem 281, 35289-35295

24. Culhane JC, Szewczuk LM, Liu X, Da G, Marmorstein R and Cole PA (2006) A mechanism-based inactivator for histone demethylase LSD1. J Am Chem Soc 128, 4536-4537

25. Huang J, Sengupta R, Espejo AB et al (2007) p53 is regulated by the lysine demethylase LSD1. Nature 449, 105-108

26. Baek SH and Kim KI (2016) Regulation of HIF-1 $\alpha$ stability by lysine methylation. BMB Rep 49, 245-246

27. Lee JY, Park JH, Choi HJ et al (2017) LSD1 demethylates HIF1 $\alpha$ to inhibit hydroxylation and ubiquitin-mediated degradation in tumor angiogenesis. Oncogene 36, 5512-5521

28. Peng B, Wang J, Hu Y et al (2015) Modulation of LSD1 phosphorylation by CK2/WIP1 regulates RNF168-dependent 53BP1 recruitment in response to DNA damage. Nucleic Acids Res 43, 5936-5947

29. Peng B, Shi R, Jiang W et al (2017) Phosphorylation of LSD1 by PLK1 promotes its chromatin release during mitosis. Cell Biosci 7, 15

30. Nam HJ, Boo K, Kim D et al (2014) Phosphorylation of LSD1 by PKC $\alpha$ is crucial for circadian rhythmicity and phase resetting. Mol Cell 53, 791-805

31. Wirtz S, Popp V, Kindermann M et al (2017) Chemically induced mouse models of acute and chronic intestinal inflammation. Nat Protoc 12, 1295-1309

32. Feng J, Xu G, Liu J et al (2016) Phosphorylation of LSD1 at Ser112 is crucial for its function in induction of EMT and metastasis in breast cancer. Breast Cancer Res Treat 159, 443-456

33. Boulding T, McCuaig RD, Tan A et al (2018) LSD1 activation promotes inducible EMT programs and modulates the tumour microenvironment in breast cancer. Sci Rep 8, 73

34. Farhadi A, Keshavarzian A, Ranjbaran Z, Fields JZ and Banan A (2006) The role of protein kinase $C$ isoforms in modulating injury and repair of the intestinal barrier. J Pharmacol Exp Ther 316, 1-7

35. Yang GJ, Lei PM, Wong SY, Ma DL and Leung CH (2018) Pharmacological inhibition of LSD1 for cancer treatment. Molecules 23, 3194

36. Fu X, Zhang P and Yu B (2017) Advances toward LSD1 inhibitors for cancer therapy. Future Med Chem 9, 12271242 